\title{
Clinical Study of Primigravida With Unengaged Head in Labour at Term
}

\author{
Priya Agarwal $^{1}{ }^{\circledR}$, Shraddha Shetty ${ }^{1 * \mathbb{D}}$
}

\begin{abstract}
Objectives: To study the effect of the unengaged fetal head in primigravida on labour outcomes and to determine its association with maternal and perinatal outcomes.

Materials and Methods: A hospital-based prospective study was conducted between August 2017 and September 2019 at hospitals affiliated with Kasturba Medical College, Mangalore. All primigravidas with an unengaged head at term with single live intrauterine gestation and vertex presentation with no cephalo pelvic disproportion were included in the study. The time of engagement, engagement to delivery interval, duration of labour, and oxytocin augmentation with the number of "fifths" of the fetal head above the pelvic brim were analyzed based on the aim of the study.

Results: A total of 350 women were evaluated in this study. The free-floating fetal head, 4/5th palpable fetal head, and 3/5th palpable were observed in $251(71.7 \%), 65(18.6 \%)$, and 34 (9.7\%) subjects, respectively. In addition, $270(77.14 \%)$ women were in the gestational period of 38-40 weeks and 191 (76.1\%) of them had the fetal head level above the brim at 38-40 weeks of gestation. Among the freefloating head, $205(68.1 \%)$ and $62(95.4 \%)$ cases in the 4/5th level of fetal head engaged in the active phase of labour and were found to be statistically significant $(P=0.0001)$. Further, the duration of labour was more than 6 hours in $159(67.1 \%)$ in the free-floating head and $39(60 \%)$ in the $4 / 5$ th level of the fetal head $(P=0.000)$. Finally, $109(43.4 \%)$ of women with the free-floating head delivered by cesarean section $(P=0.087)$.

Conclusions: In general, primigravida with an unengaged fetal head at term in labour requires intense monitoring, intervention, and labour augmentation in order to reduce the maternal morbidity and perinatal complications.

Keywords: Primigravida, Unengaged head, Labour, Free-floating head, Augmentation
\end{abstract}

\section{Introduction}

Labour begins with uterine contraction and ends with the delivery of newborns and expulsion of the placenta $(1,2)$. According to (3), the left occipitoanterior position is more common than the right occipitoanterior as the left oblique diameter is encroached by the rectum. In addition, the engaging anteroposterior diameter of the head is suboccipitobregmatic $(9.5 \mathrm{~cm})$ and the transverse diameter is biparietal $(9.5 \mathrm{~cm})$.

The descent is a continuous process, slow in the first stage but pronounced in the second stage provided that there is no undue bony or soft tissue obstruction. The progressive descent of the head is abdominally assessed by estimating the number of "fifths" of the head above the pelvic brim (Crichton). Further, 5/5th of the head is floating above the brim, $4 / 5$ th if the head is on the brim, and $3 / 5$ th if the head has a major part in the brim. Once the widest diameter has passed the brim, the head is assumed to be $2 / 5$ palpable, and it is engaged or both poles of the fetal head (i.e., sinciput and occiput) are palpable by the 2 nd pelvic grip. Internal examinations reveal the descent of the head to ischial spines and the position of the head (3).
In primigravidas, engagement occurs in a significant number of cases before the onset of labour by around 38 weeks while in multiparas, the same procedure may occur in the late first stage with the rupture of the membrane. It is noteworthy that cephalopelvic disproportion should be ruled out in a primigravida with the nonengagement of the head even at labour (3). Furthermore, nulliparous women at term with a floating head are at the increased risk of cesarean section for abnormal labour. However, the majority of patients will still deliver vaginally (4). Moreover, primigravidas with an unengaged fetal head at term are considered as high-risk cases (5).

\section{Objectives of the Study}

The present study aimed to investigate the effect of the unengaged fetal head on labour outcomes in primigravida and its association with the time of engagement, engagement to delivery interval, the duration of labour, and maternal and perinatal outcomes.

\section{Materials and Methods}

A hospital-based prospective study was performed in the hospitals affiliated with Kasturba Medical College, 
Mangalore between August 2017 and September 2019. All primigravidas with an unengaged head at term (between 37 and 42 weeks of gestation) in labour with single live intrauterine gestation and vertex presentation, no obvious cephalopelvic disproportion, and membrane intact were included in the study. Then, 350 women fulfilling the inclusion criteria were included in the study. Informed written consent was taken from the participants and institutional ethical committee clearance was obtained as well.

\section{Exclusion Criteria}

Primigravida $>35$ years age, fetal anomalies, fibroid, ovarian tumors, placenta previa, and medical complications like diabetes mellitus, hypertension, thyroid disorders, cardiac disease, and epilepsy.

\section{Materials and Methods}

All women admitted to the labour room were included in this study, and demographic details were noted in a pretested proforma. The fetal head was considered unengaged when more than 2/5th of this head was palpable on abdominal examinations (the Crichton's method) or both poles of the fetal head (i.e., sinciput and occiput) were palpable by the 2 nd pelvic grip.

The per-vaginal examination was done and then cervical consistency, position, effacement and dilatation (in $\mathrm{cm}$ ), the station of the fetal head, and the status of the membrane were noted as well. In addition, the Muller Munro Kerr maneuver was used to rule out cephalopelvic disproportion, followed by measuring the diagonal conjugate. Similarly, admission cardiotocography was taken and a partogram was plotted to assess the course and duration of labour. Further, the maternal outcome was evaluated by the time of engagement, engagement to delivery interval, the duration of labour, augmentation with oxytocin, and the mode of delivery. Finally, the neonatal outcome was assessed by the Apgar score at 1 minute and 5 minutes.

\section{Statistical Analysis}

The SPSS software, version 23 was used for data analysis, and a $P$ value of less than 0.05 was considered to be statistically significant. Eventually, chi-square and Fischer's exact tests were applied to analyze the results.

\section{Results}

In general, 350 women were studied for two years. The fetal head was $5 / 5$ th, $4 / 5$ th, and $3 / 5$ th palpable in $251(71.7 \%)$, $65(18.6 \%)$, and $34(9.7 \%)$ participants, respectively. The unengaged fetal head was observed in most primigravidas in the age group of 20-30 years. Furthermore, most of the primigravida had an unengaged fetal head within the gestational age of 38-40 weeks (Table 1). Moreover, 232 (66.2\%) of the primigravida presented in the latent phase of labour with the unengaged fetal head. Additionally, the

\section{Key Messages}

- Primigravida with an unengaged fetal head at term is an obstetric risk factor.

- Cephalopelvic disproportion has to be ruled to reduce the incidence of cervical dystocia and obstructed labour

- Timely intervention and intense monitoring is necessary to reduce maternal and perinatal complications

engagement occurred in the active phase of labour in 301 $(86 \%)$ of primigravida with an unengaged fetal head at all fifths above the pelvic brim and the results were found to be statistically significant. Similarly, the unengaged fetal head was observed in $14(5.6 \%)$ primigravida with a free-floating head. In the majority of the unengaged heads in labour, engagement to the delivery interval was 6-10 hours and was statistically significant. In addition, the mean duration of labour was 12.99 hours in the freefloating head, suggesting that labour is prolonged with higher levels of the fetal head. Primi with unengaged heads in labour required oxytocin augmentation for the progression of labour (Table 2).

Among 251 women with a free-floating head, 140 (55.7\%) cases delivered vaginally, $2(0.8 \%)$ of them had assisted vaginal delivery, and 109 (43.42\%) cases underwent a cesarean section (C-section). In the $4 / 5$ th level of the fetal head, $43(66.2 \%)$ and $22(33.8 \%)$ of women delivered vaginally and underwent a C-section, respectively. In the 3/5th level of the fetal head, 26 (76.5\%) and $8(23.5 \%)$ cases delivered vaginally and underwent a C-section, respectively, and the result was statistically significant (Table 3). These observations suggest that the mode of delivery is determined by the level of the fetal head. The C-section rate was higher with a free-floating head. Further, the most common indication for the C-section was the failure to progress labour in all fifths of the fetal head above the pelvic brim, and the results were reported to be statistically significant (Table 4). Based on the results, the birth weight did not change with different levels of the unengaged fetal head, and no statistically

Table 1. Demographic Characteristics

\begin{tabular}{lccc}
\hline & \multicolumn{3}{c}{ Fifth of Fetal Head Palpable per Abdomen } \\
\cline { 2 - 4 } Characteristics & $\mathbf{5 / 5}$ & $\mathbf{4 / 5}$ & $\mathbf{3 / 5}$ \\
& $\mathbf{n ~ ( \% )}$ & $\mathbf{n}(\mathbf{\%})$ & $\mathbf{n ~ ( \% )}$ \\
\hline Age & & & \\
$<20$ & $24(9.6)$ & $6(9.23)$ & $6(17.6)$ \\
$20-25 s$ & $110(43.8)$ & $26(40)$ & $12(35.3)$ \\
$26-30$ & $97(38.6)$ & $31(47.7)$ & $11(32.4)$ \\
$>30$ & $20(7.9)$ & $2(3.1)$ & $5(14.7)$ \\
Total & 251 & 65 & 34 \\
Gestational age (wk) & & & \\
$37-38$ & $27(10.8)$ & $7(10.8)$ & $6(17.6)$ \\
$38-40$ & $191(76.1)$ & $52(80)$ & $27(79.4)$ \\
$40-42$ & $33(13.1)$ & $6(2.4)$ & $1(2.9)$ \\
\hline
\end{tabular}


Table 2. Labour Outcome in Unengaged Fetal Head

\begin{tabular}{|c|c|c|c|c|}
\hline \multirow{3}{*}{ Labour Outcome } & \multicolumn{3}{|c|}{ Fifth of Fetal Head Palpable } & \multirow{3}{*}{$P$ Value } \\
\hline & $5 / 5$ & $4 / 5$ & $3 / 5$ & \\
\hline & n (\%) & n $(\%)$ & n (\%) & \\
\hline \multicolumn{5}{|l|}{ Stage of labour } \\
\hline Latent phase & $170(67.7 \%)$ & $44(67.7 \%)$ & $18(52.9 \%)$ & \multirow{2}{*}{$0.0001(\mathrm{HS})$} \\
\hline Active phase & $81(32.3 \%)$ & $21(32.3 \%)$ & $16(47.1 \%)$ & \\
\hline \multicolumn{5}{|l|}{ Time of engagement } \\
\hline Latent phase & $32(13.5 \%)$ & $3(4.62 \%)$ & $0(0)$ & \multirow{3}{*}{$0.0001(\mathrm{HS})$} \\
\hline Active phase & $205(86.5 \%)$ & $62(95.4 \%)$ & $34(100 \%)$ & \\
\hline Unengaged & $14(5.6 \%)$ & $0(0)$ & $0(0)$ & \\
\hline \multicolumn{5}{|c|}{ Engagement to delivery interval } \\
\hline$<2$ hours & $0(0)$ & $0(0)$ & $5(14.7 \%)$ & \multirow{4}{*}{$0.0003(\mathrm{HS})$} \\
\hline 2-6 hours & $76(32.3 \%)$ & $26(40 \%)$ & $14(41.2 \%)$ & \\
\hline 6-10 hours & $127(53.6 \%)$ & $35(53.8 \%)$ & $16(47.1 \%)$ & \\
\hline$>10$ hours & $34(14.5 \%)$ & $4(6.2 \%)$ & $0(0)$ & \\
\hline Mean duration of labour & 12.99 hours & 8.22 hours & 5.77 hours & $0.000(\mathrm{HS})$ \\
\hline Oxytocin augmentation & $202(80.5 \%)$ & $47(72.3 \%)$ & $22(64.7 \%)$ & $0.000(\mathrm{HS})$ \\
\hline
\end{tabular}

Note. HS: Highly significant.

Table 3. Mode of Delivery in Relation to Fetal Head Levels

\begin{tabular}{|c|c|c|c|c|}
\hline \multirow{2}{*}{ Mode of Delivery } & \multicolumn{3}{|c|}{ Fifth of Fetal Head Palpable } & \multirow[t]{2}{*}{$P$ Value $^{*}$} \\
\hline & $5 / 5$ & $4 / 5$ & $3 / 5$ & \\
\hline Vaginal & $140(55.7)$ & $43(66.2)$ & $26(76.5)$ & $0.000(\mathrm{HS})$ \\
\hline Assisted vaginal delivery & $2(0.8)$ & $0(0)$ & $0(0)$ & 0.087 (NS) \\
\hline LSCS & $109(43.42)$ & $22(33.8)$ & $8(23.5)$ & $0.000(\mathrm{HS})$ \\
\hline
\end{tabular}

Note. LSCS: Lower segment caesarean section. ${ }^{*} P<0.05$ is significant; HS: Highly significant; NS: Not significant.

Table 4. Indication for LSCS

\begin{tabular}{lccc}
\hline \multirow{2}{*}{ Mode of Delivery } & \multicolumn{2}{c}{ Fifth of Fetal Head Palpable $^{*} \boldsymbol{P}$ Value } \\
\cline { 2 - 3 } & $\mathbf{5 / 5}$ & $\mathbf{4 / 5}$ & $\mathbf{3 / 5}$ \\
\hline Failure to progress of labour & $74(67.9 \%)$ & $16(72.7 \%)$ & $4(50 \%)$ \\
Deep transverse arrest & $3(2.75 \%)$ & $1(4.54 \%)$ & $0.000(\mathrm{HS})$ \\
Fetal distress & $32(29.35 \%)$ & $5(22.7 \%)$ & $0.000(\mathrm{HS})$ \\
\hline
\end{tabular}

Note. LSCS: Lower segment caesarean section. ${ }^{*} P<0.05$ is significant; HS: Highly significant; NS: Not significant.

Table 5. Perinatal Outcome in Unengaged Head

\begin{tabular}{|c|c|c|c|c|c|c|c|}
\hline \multirow{4}{*}{$\begin{array}{l}\text { Perinatal Outcome } \\
\text { Mean birth weight }\end{array}$} & \multicolumn{6}{|c|}{ Fifth of Fetal Head Palpable } & \multirow{2}{*}{$P$ Value } \\
\hline & \multicolumn{2}{|c|}{$5 / 5$} & \multicolumn{2}{|c|}{$4 / 5$} & \multicolumn{2}{|c|}{$3 / 5$} & \\
\hline & Weight(kg) & SD & Weight(kg) & SD & Weight(kg) & SD & \\
\hline & 2.83 & 0.40 & 2.78 & 0.42 & 2.91 & 0.48 & NS \\
\hline \multicolumn{8}{|l|}{ APGAR 1 minute } \\
\hline$>7$ & \multicolumn{2}{|c|}{$228(90.8 \%)$} & \multicolumn{2}{|c|}{$62(95.4 \%)$} & \multicolumn{2}{|c|}{$33(97.1 \%)$} & NS \\
\hline$<7$ & \multicolumn{2}{|c|}{$23(9.2 \%)$} & \multicolumn{2}{|c|}{$3(4.6 \%)$} & \multicolumn{2}{|c|}{$1(2.9 \%)$} & \\
\hline
\end{tabular}

Note. APGAR: Appearance, pulse, grimace, activity, and respiration; SD: Standard deviation; NS: Not significant.

significant changes were observed in the Apgar score with the levels of the unengaged fetal head (Table 5).

The results further revealed that maternal complications like the cervical tear, the perineal tear, and atonic postpartum hemorrhage were observed in 17 (6.7\%), 12 (4.7\%), and $32(12.7 \%)$ free-floating heads. Furthermore, the rate of maternal complications was higher in the freefloating head compared to the $4 / 5$ th and $3 / 5$ th levels of the head (Table 6).

\section{Discussion}

In the present study, the total sample size was 350 . Moreover, the fetal head was $5 / 5$ th, $4 / 5$ th, and $3 / 5$ th palpable in 251 (71.7\%), 65 (18.6\%), and 34 (9.7\%) women, respectively, which is in line with the results of other studies $(6,7)$.

The majority of the primigravida had a free-floating head at term. Ansari et al conducted a study on 100 primigravidas aged $17-30$ years at term and found that $58 \%$ 
Table 6. Maternal Complications

\begin{tabular}{lccc}
\hline \multirow{2}{*}{ Maternal Complications } & \multicolumn{3}{c}{ Fifth of Fetal Head Palpable } \\
\cline { 2 - 4 } & $\mathbf{5 / 5}$ & $\mathbf{4 / 5}$ & $\mathbf{3 / 5}$ \\
\hline Cervical tear & $17(6.7 \%)$ & $2(3.1 \%)$ & 0 \\
Perineal tear & $12(4.7 \%)$ & $3(4.6 \%)$ & $1(2.9 \%)$ \\
Atonic postpartum hemorrhage & $32(12.7 \%)$ & $2(3.1 \%)$ & $1(2.9 \%)$ \\
\hline
\end{tabular}

had a free-floating fetal head. Additionally, cephalopelvic disproportion was the most common cause of the freefloating head, followed by malposition, macrosomia, placenta previa, a cord around the fetal neck, and uterine fibroid (3). In another study, the deflexed head was the commonest cause of the unengaged head in primigravida, and no cause was identified in 60 (40\%) of subjects (6).

In the present study, most primigravida with an unengaged head in labour were in the age group of 20-30 years. Mahendra and Prameela focused on 130 primigravidas with an unengaged head at term in labour and observed that most women were of the age group of 21-25 years (7).

The unengaged fetal head was observed in the gestational age of 38-40 weeks in most primigravida. Engagement occurs by 38 weeks in all the primigravida. The causes of the non-engagement of the head are the deflexed head, cephalopelvic disproportion, fetal malposition, macrosomia, and soft tissue masses in the pelvis, including uterine fibroids, ovarian tumors, and placenta previa (5-7).

The engagement occurred in the active phase of labour in 301 (86\%) of the primigravida with an unengaged fetal head, and the head did not engage in 14 (5.6\%) of subjects with a free-floating head. In a similar study conducted by Mahendra and Prameela, the head engaged in 93.2\% and $4.6 \%$ in active and latent labour, respectively, while unengaged in $3.75 \%$ of women $(7,8)$.

In the majority of the unengaged heads, engagement to the delivery interval lasted 6-10 hours, and the mean duration of labour was 12.99 hours in the free-floating head, suggesting that the progression of the labour is associated with the level of the fetal head during the labour. In addition, primi with unengaged head in labour required oxytocin augmentation for the progression of labour. Further, the mean duration of labour was more in the free-floating head in comparison to the $4 / 5$ th and $3 / 5$ th levels of the fetal head. Based on the results of a study by Pahwa et al, the need for the augmentation of labour with oxytocin was more remarkable in higher head stations (9). Mahajan et al also observed that $90 \%$ of women required augmentation with oxytocin with an unengaged head (9). Similar results were noted in another study (6).

In the present study, 140 (55.7\%) women delivered vaginally and $2(0.8 \%)$ of them had forceps assisted vaginal delivery. Furthermore, 109 (43.42\%) of women underwent a C-section in the free-floating head, representing that the cesarean delivery rate is higher with a free-floating head.
The results further revealed that 139 (39.2\%) of subjects with unengaged heads underwent a C-section. The most common indication was the failure of the progress of labour, followed by fetal distress and deep transverse arrest. In their study, Pahwa et al reported that $56 \%, 8 \%$, and $36 \%$ of women delivered by a C-section, forceps assisted vaginal delivery, and normal vaginal delivery, respectively (9).

Based on the results of the present study, the birth weight did not change with different levels of the unengaged fetal head. However, it was found that mean birth weight was $2.84 \pm 0.31$ and it was more in higher fetal head levels in comparison to lower levels. Moreover, the Apgar score at 1 minute was less than 7 in 23 (9.2\%) women in the freefloating head, and it was observed that the higher level of the fetal head increases perinatal morbidity. In a study by Neha et al, perinatal mortality was documented in one case of forceps delivery in the free-floating head (8).

The findings of the study demonstrated that atonic postpartum hemorrhage was the commonest maternal complication in $32(12.7 \%)$ cases, followed by the cervical and perineal tear in 17 (6.7\%) and $12(4.7 \%)$ women with free-floating heads, respectively. The rate of maternal complications was higher in the free-floating head compared to the $4 / 5$ th and 3/5th levels of the head. Similar maternal complications were reported in other studies (8-11). Some studies focusing on primigravida with unengaged heads in labour found that unengaged heads in primigravida in labour is not an indication for lower segment $\mathrm{C}$-section and concluded that timely intervention increases the chance of vaginal delivery, and thus reducing maternal and perinatal morbidity (12-14). Likewise, Hashim et al conducted a comparative casecontrol study at Sir Syed Hospital in Karachi and observed that Primigravidas were at higher risk of prolonged labour, fetal distress during labour, operative vaginal delivery, and emergency C-section (15). In another study, Iqbal et al compared two groups of Primigravida cases with unengaged $(n=100)$ and engaged $(n=100)$ heads. In the unengaged group, $62 \%$ and $38 \%$ had a normal vaginal delivery and a cesarean delivery, respectively, and $85 \%$ had normal vaginal delivery in the engaged group. They concluded that primigravidas with the unengaged fetal head at term or the onset of labour need intense monitoring for a reduction in the perinatal and maternal morbidity (16).

Sudhir and Mishra studied 20 primigravida cases with an unengaged head at term at the onset of labour and observed that the first and second stages of labour lasted for 7-10 hours and 55-110 minutes, respectively. Additionally, 78 women had a vaginal delivery while 42 cases underwent a $\mathrm{C}$-section. In vaginal delivery, forceps delivery and vacuum-assisted vaginal delivery were noted in 14 and 6 cases, respectively. Failure to progress labour was the most common indication for cesarean delivery. They concluded that vaginal delivery is possible in a 
primigravida with an unengaged head at labour at term with watchful expectancy and the usage of appropriate means of the intervention (17). Similar results were observed in another study (18).

Burke et al did a multicenter genesis study on term nulliparous women. Five parameters (i.e., maternal age, body mass index, height, fetal abdominal circumference, and fetal head circumference) were used to determine the risk of cesarean delivery. Based on their results, advancing maternal age, shorter maternal height, increasing body mass index, larger fetal abdominal circumference, and larger fetal head circumference increased the risk of cesarean delivery. Finally, they found that the risk tool may be used to predict the success rate at achieving an uncomplicated vaginal delivery or planned cesarean delivery (19).

In a similar study, Eggebo et al evaluated 122 nulliparous women in the first stage of labour. To this end, transabdominal ultrasound was used to determine the position of the fetal head and digital examination for cervix dilatation, as well as to assess the head-perineum distance and caput succedaneum. A risk score was obtained with a vaginal delivery as the outcome, which was dichotomized into cesarean and vaginal delivery. They concluded that vaginal delivery can be predicted based on maternal characteristics and findings during labour (20).

\section{Conclusions}

In general, primigravida with an unengaged head at term in labour are vulnerable for higher cesarean section rates. Thus, labour should be managed with intense care and vigilant monitoring, and necessary interventions like partogram, cardiotocography, labour augmentation, and instrumental delivery facilitate vaginal delivery and reduce maternal and perinatal morbidity and mortality.

\section{Authors' Contribution}

PA: Study concept and design, Data analysis and interpretation and Statistical analysis. SS: Study concept and design, Study supervision, Critical revision of the manuscript, Manuscript editing and drafting.

\section{Conflict of Interests}

Authors declare that they have no conflict of interests.

\section{Ethical Issues}

The present study was approved by the Institutional Ethics Committee of Kasturba Medical College, Mangalore (IEC KMC MLR 08-17/147).

\section{Financial Support}

This study received no financial support.

\section{Acknowledgments}

The authors would like to thank the Medical Superintendent of Lady Goschen Hospital and Kasturba Medical College Hospital, Attavar, Mangalore for permitting to carry out the study.

\section{References}

1. Cunningham FG, Leveno KJ, Bloom SL, Hauth JC, Gilstrap III LC, Wenstrom KD. Williams Obstetrics. 22nd ed. New York: McGrawHill; 2005.

2. Rouse DJ, St John E. Normal Labor, Delivery, Newborn Care, and Puerperium. 9th ed. Philadelphia: Lippincott Williams \& Wilkins; 2003:35-37.

3. Dutta DC. Normal Labour. 8th ed. New Delhi: Jaypee Brothers; 2015:134-138. Chapter 13

4. Debby A, Rotmensch S, Girtler O, Sadan O, Golan A, Glezerman M. Clinical significance of the floating fetal head in nulliparous women in labor. J Reprod Med. 2003;48(1):37-40.

5. Chaudhary S, Farrukh R, Dar A, Humayun S. Outcome of labour in nullipara at term with unengaged vertex. J Ayub Med Coll Abbottabad. 2009;21(3):131-134.

6. Ansari A, Akhtar S, Aamir M. Causes and management of high fetal head in primigravidae at term. Pak Armed Forces Med J. 2008;58(1):16-20.

7. Mahendra G \& Prameela. Clinical Study of Un-Engaged Head in Primigravida at Term in Labour. International Journal of Recent Trends in Science and Technology. 2014;10:345-349.

8. Mahajan N, Mustafa S, Tabassum S, Fareed P. Outcome of high fetal station in primigravida at term in labour. Int J Reprod Contracept Obstet Gynecol. 2016;5(3):873-877. doi:10.18203/2320-1770. ijrcog20160602

9. Pahwa S, Kaur A, Nagpal M. Obstetric outcome of floating head in primigravida at term. Int J Reprod Contracept Obstet Gynecol. 2018;7(1):242-247. doi:10.18203/2320-1770.ijrcog20175854

10. Shrivastava A. Obstetrical outcome in Primigravidas with unengaged fetal head at spontaneous onset of labour at term gestation. Int J Sci Res. 2017;6(8):3-4. doi:10.36106/ijsr

11. Banu DS, Sultana $R$ Khatun $M$, et al. Maternal outcome of among the primigravida women presented with fetal head engagement: experience of 1440 cases in Bangladesh. J Sci Found. 2018;16(2):34-37. doi:10.3329/jsf.v16i2.40866

12. Unnisa S, Poornima MS. Unengaged head at term in Primigravida: does it affect the chance of having a normal delivery? Int J Reprod Contracept Obstet Gynecol. 2019;8(4):1616-1619. doi:10.18203/2320-1770.ijrcog20191228

13. Shaikh F, Shaikh S, Shaikh N. Outcome of primigravida with high head at term. J Pak Med Assoc. 2014;64(9):1012-1014.

14. Ambwani BM. Primigravidas with floating head at term or onset of labor. Int J Gynecol Obstet. 2004;3(1):1.

15. Hashim N, Naqvi S, Khanam M, Jafry HF. Primiparity as an intrapartum obstetric risk factor. J Pak Med Assoc. 2012;62(7):694698.

16. Iqbal S, Sumaira S. Outcome of primigravidae with unengaged versus engaged fetal head at term or onset of labour. Biomedica. 2009;25:159-162.

17. Sudhir S, Mishra S. The outcome of labour in primigravida with term gestation and unengaged head at onset of labour. Indian J Obstet Gynecol Res. 2016;3(3):199-202. doi:10.5958/23942754.2016.00045.x

18. Oboro VO, Tabowei TO, Bosah JO. Fetal station at the time of labour arrest and risk of caesarean delivery. J Obstet Gynaecol. 2005;25(1):20-22. doi:10.1080/01443610400022512

19. Burke N, Burke G, Breathnach F, et al. Prediction of cesarean delivery in the term nulliparous woman: results from the prospective, multicenter Genesis study. Am J Obstet Gynecol. 2017;216(6):598. e591-598.e511. doi:10.1016/j.ajog.2017.02.017

20. Eggebø TM, Wilhelm-Benartzi C, Hassan WA, Usman S, Salvesen KA, Lees CC. A model to predict vaginal delivery in nulliparous women based on maternal characteristics and intrapartum ultrasound. Am J Obstet Gynecol. 2015;213(3):362.e361-366. doi:10.1016/j.ajog.2015.05.044 